\title{
Combination of treadmill exercise with bone marrow stromal cells transplantation activates protein synthesis-related molecules in soleus muscle of the spinal cord injured rats
}

\author{
You-Mi Kim', Eun-Sang Ji ${ }^{2}$, Il-Gyu Ko ${ }^{2}$, Jun-Jang Jin² , Yeong-Hyun Cho ${ }^{3}$, Tae-Beom Seo ${ }^{3, *}$ \\ ${ }^{1}$ Sports Science Research Institution, Korea National Sport University, Seoul, Korea \\ 2Department of Physiology, College of Medicine, Kyung Hee University, Seoul, Korea \\ ${ }^{3}$ Department of Kinesiology, College of Natural Science, Jeju National University, Jeju, Korea
}

The present study investigated whether treadmill exercise with bone marrow stromal cells (BMSCs) transplantation increase expression level of protein synthesis-related molecules in the soleus muscle after spinal cord injury (SCI). The spinal cord contusion injury was performed at the T9-10 level using the impactor $(10 \mathrm{~g} \times 25 \mathrm{~mm})$. BMSCs were cultured from femur and tibia of 4-week-old rats and then transplanted directly into the lesion 1-week post injury. The rats in exercise group were walking on treadmill device for 6 days per a week during 6 weeks. Prepared soleus muscles were used for examining mechanisms of protein synthesis after SCl. Myostatin induction level was increased by SCl, but BMSCs engrafting after SCI decreased compared to SCI group. Combination of treadmill exercise with BMSCs showed more potent decrement on myostatin expression. Protein kinase B (Akt) and mammalian target of rapamycin (mTOR) levels were significantly increased in SCI and BMSCs transplantation group compared to SCI group. Combination of treadmill exercise with BMSCs further facilitated expression levels of Akt and mTOR. Insulin-like growth factor-I (IGF-I) and phosphorylated cyclic adenosine monophosphate response element-binding protein ( $p$-CREB) induction levels were more increased in SCI and BMSC transplantation group compared to $\mathrm{SCI}$ group. Combination of treadmill exercise with BMSCs further increased expression levels of IGF-I and $\mathrm{p}$-CREB, although statistical significance was not appeared. Combining treadmill exercise with BMSCs transplantation might accelerate protein synthesis and hypertrophy in the soleus muscle after SCI through activation of IGF-I/mTOR signaling pathway.

Keywords: Spinal cord injury, Treadmill exercise, Bone marrow stromal cells, Myostatin

\section{INTRODUCTION}

After spinal cord injury (SCI), astrocytes and olygodencrocytes around the injury area secrete inhibitory substrates to prevent the regeneration, leading to reactive astrocyte formation, glial scar, axonal dieback, demyelination in the injured spinal cord (DePaul et al., 2017; Thompson and Sakiyama-Elbert, 2018). For the success of spinal cord regeneration, cell transplantation therapy using neural stem cells, Schwann cells, fibroblasts, and bone marrow stromal cells (BMSCs) has been attempted (Ide et al., 2010; Ou- dega and $\mathrm{Xu}, 2006)$.

In particular, BMSCs do not have side effect and ethical problem because they are extracted from the patient's bone marrow. Engrafting BMSCs could improve neuroplasticity and motor functional recovery of the injured spinal cord through up-regulation of brain-derived neurotropic factor (BDNF)-tyrosine kinase B receptor signaling pathway (Tu et al., 2018). BDNF and nerve growth factor in the central and peripheral nervous system are activated by regular low- and middle-intensity exercise (Jung et al., 2016; Tian et al., 2018). In the field of spinal cord regeneration,

\footnotetext{
${ }^{*}$ Corresponding author: Tae-Beom Seo (i) https://orcid.org/0000-0001-9213-2251 Department of Kinesiology, College of Natural Science, Jeju National University, 102 Jejudaehak-ro, Jeju 63243, Korea

E-mail: seotb@jejunu.ac.kr

Received: May 2, 2019 / Accepted: June 3, 2019
}

This is an Open Access article distributed under the terms of the Creative Commons Attribution Non-Commercial License (http://creativecommons.org/licenses/by-nc/4.0/) which permits unrestricted non-commercial use, distribution, and reproduction in any medium, provided the original work is properly cited. 
voluntary physical activity increased locomotor functions after SCI through activation of neurotrophic factors into the injury site (Seo et al., 2013; Wheaton et al., 2013).

Decreased physical activity by SCI up-regulates the expression of the atrophy-related proteins and down-regulates the ability to use oxygen in the skeletal muscle, and then leads to loss of functional capacity (Ibitoye et al., 2016). For inhibition of skeletal muscle atrophy by SCI, cell transplantation therapy and physical activity have been applied in spinal cord injured rats and patients.

Regenerative mechanism of the injured spinal cord by cell engrafting or exercise has been well documented, but the synergistic effect of combined cell transplantation and exercise on prevention of the muscle atrophy after SCI is still unclear. Therefore, the present study investigated whether treadmill exercise combined with BMSCs transplantation increased activation of protein synthesisrelated signaling pathway, myostatin, protein kinase B (Akt), mammalian target of rapamycin (mTOR), insulin-like growth factor-I (IGF-I), phosphorylated cyclic adenosine monophosphate response element-binding protein (p-CREB), in the soleus muscle after SCI.

\section{MATERIALS AND METHODS}

\section{Animals and treatments}

Forty adult male Sprague-Dawley rats (180 \pm 10 g, 6 weeks old) were maintained in an animal room with a regulated temperature. The experimental procedures were performed in accordance with the animal care guidelines of the National Institute of Health and the Korean Academy of Medical Sciences. The animals were randomly divided into four groups ( $\mathrm{n}=10$ in each group): control group, SCI group, SCI and BMSCs transplantation group, and SCI and BMSCs transplantation with treadmill exercise group.

\section{Spinal cord contusion injury}

Spinal cord contusion injury was induced according to the previously described method (Kim et al., 2017). The rats were anesthetized with Zoletil $50(10 \mathrm{mg} / \mathrm{kg}$, intraperitoneally; Vibac Laboratories, Carros, France). A laminectomy was performed to expose the spinal cord at thoracic level T9-10 without disrupting the dura. A contusion injury was created using the New York University Impactor System (NYU impactor, New York, NY, USA) by dropping a $10-\mathrm{g}$ impactor from $2.5-\mathrm{cm}$ height onto the exposed dura.

\section{BMSCs culture and transplantation}

BMSCs were obtained by perfusion through femur and tibia of 4-week-old rats, as the previously described method (Kim et al., 2018; Shin et al., 2016). Cells were cultured in Dulbecco's modified Eagle's medium with $20 \%$ fetal calf serum. Seven days later, cultured BMSC at a density of $5 \times 10^{6}$ in $50-\mu \mathrm{L}$ phosphate-buffered saline (PBS) was injected into the cavity of the injured spinal cord using a micro syringe with a 30-gauge needle.

\section{Treadmill exercise protocol}

The rats in the exercise group were conducted daily by having the rats run at a speed of $8 \mathrm{~m} / \mathrm{min}$ on a horizontal platform for 30 min once a day, 6 days a week for 6 weeks.

\section{Western blot analysis}

The animals were fully anesthetized using Zoletil $50(10 \mathrm{mg} / \mathrm{kg}$, intraperitoneally) and then the soleus muscle was dissected. Western blot analysis was performed to investigate expression levels of atrophy- and hypertrophy-related proteins in the soleus muscle after SCI according to the previously described method (Kim et al., 2018). Bilateral soleus muscles located under the gastrocnemius were prepared and washed with ice-cold PBS. All samples were sonicated in 1,000 $\mathrm{mL}$ of Triton lysis buffer. Protein separation was performed using a $10 \%$ polyacrylamide with $0.05 \%$ bisacrylamide. Proteins were then transferred to nitrocellulose and the blots were probed with anti-myostatin mouse monoclonal antibody (1:1,000, Santa Cruz Biotechnology, Santa Cruz, CA, USA), anti-Akt rabbit polyclonal antibody (1:1,000, Cell Signaling Technology, Danvers, MA, USA), anti-p-CREB rabbit polyclonal antibody (1:1,000, Cell Signaling Technology), anti-mTOR rabbit polyclonal antibody (1:500, Cell Signaling Technology), anti-IGF-I rabbit monoclonal antibody (1:1,000, Santa Cruz Biotechnology), and anti- $\beta$-actin mouse monoclonal antibody (1:3,000, Santa Cruz Biotechnology). Peroxidase anti-rabbit IgG (1:5,000, Vector Laboratories, Burlingame, CA, USA) and peroxidase anti-mouse $\operatorname{IgG}(1: 5,000$, Vector Laboratories) were used as the secondary antibodies. Immunoreactivity was detected by enhanced chemiluminescence (Santa Cruz Biotechnology).

\section{Data analysis}

The detected bands were calculated densitometrically using Molecular Analyst, version 1.4.1 (Bio-Rad, Hercules, CA, USA). Statistical analysis was performed using one-way analysis of variance followed by Duncan post boc test, and the results were expressed as the mean \pm standard error of the mean. Significance was set as $P<0.05$. 


\section{RESULTS}

\section{Changes of myostatin expression level}

The expression level of myostatin in soleus muscle of the injured spinal cord is presented in Fig. 1. Myostatin induction level was increased by SCI, but BMSCs engrafting after SCI decreased compared to SCI group. In particular, combination of treadmill exercise with BMSCs showed more potent decrement on myostatin expression, suggesting that treadmill exercise combined with BMSCs helps to reduce skeletal muscle atrophy of the injured spinal cord.

\section{Changes of Akt and mTOR levels}

The expression levels of Akt and mTOR in the injured spinal cord are presented in Fig. 2. Both Akt and mTOR levels were significantly increased in SCI and BMSCs transplantation group compared to SCI group. Combination of treadmill exercise with BMSCs further facilitated expression levels of Akt and mTOR, suggesting that treadmill exercise combined with BMSCs helps to activate hypertrophy-related protein synthesis in the skeletal muscle after SCI.

\section{Changes of IGF-I and p-CREB levels}

The expression levels of IGF-I and p-CREB in the injured spinal cord are presented in Fig. 3. IGF-I and p-CREB induction
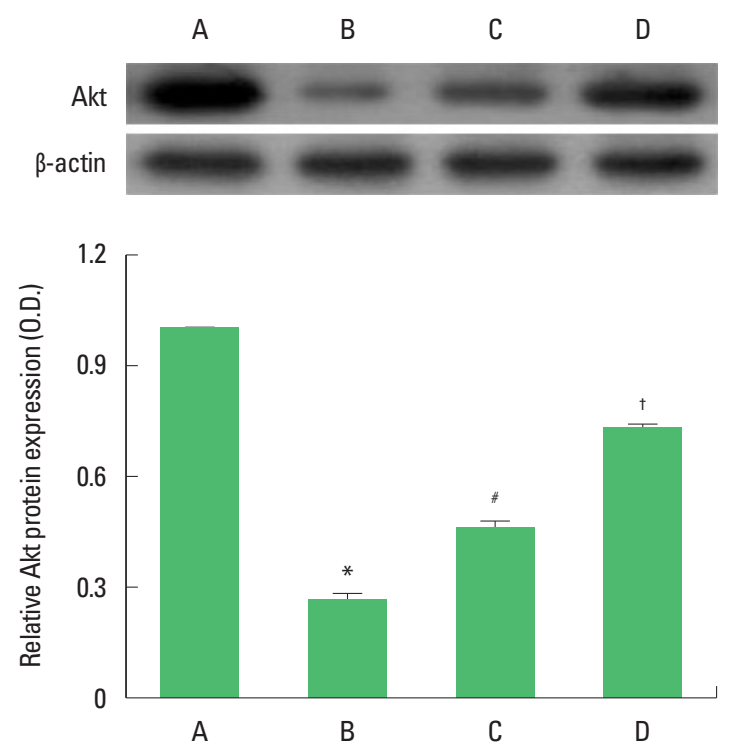

levels were more increased in SCI and BMSC transplantation group compared to SCI group. Combination of treadmill exercise with BMSCs further increased expression levels of IGF-I and

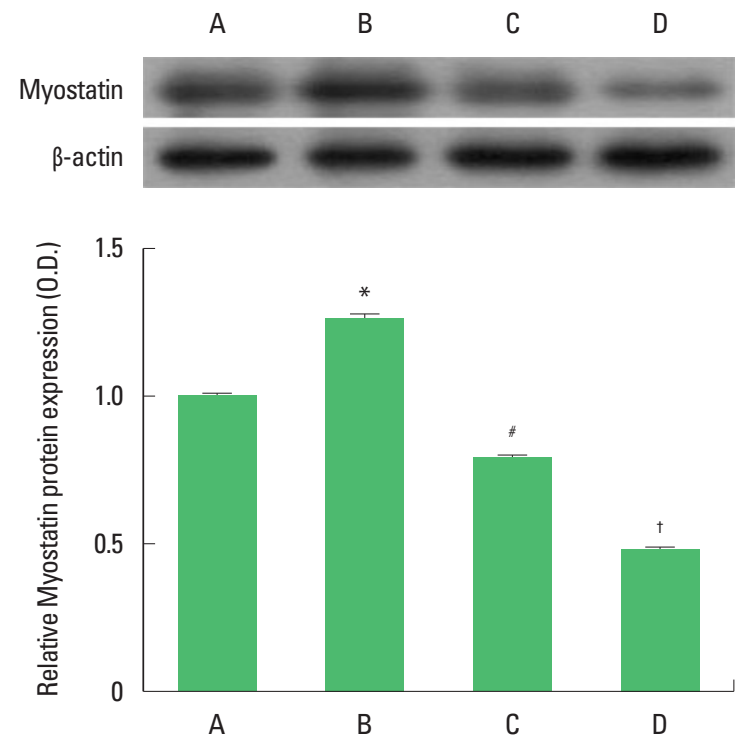

Fig. 1. Myostatin expression in the soleus muscle. Upper panel: Representative expressions of myostatin and $\beta$-actin. Lower panel: Relative myostatin expression. A, control group; B, spinal cord injury (SCI) group; C, SCl and bone marrow stromal cells (BMSCs) transplantation group; D, SCl and BMSCs transplantation with treadmill exercise group. ${ }^{*} P<0.05$ compared to control group. ${ }^{*} P<0.05$ compared to $\mathrm{SCl}$ group. ${ }^{\dagger} P<0.05$ compared to $\mathrm{SCl}$ and BMSCs transplantation group.

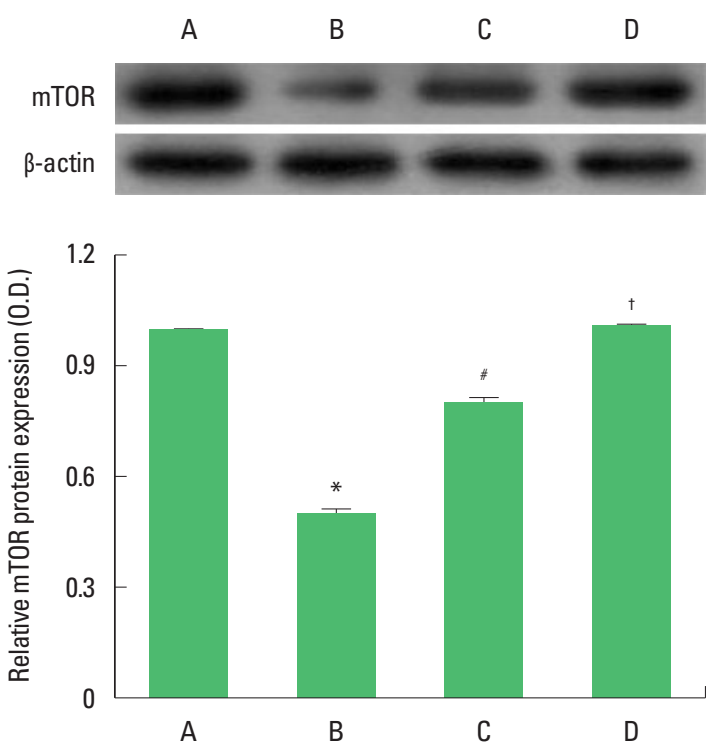

Fig. 2. Protein kinase B (Akt) and mammalian target of rapamycin (mTOR) expressions in the soleus muscle. Upper panel: Representative expressions of Akt, mTOR, and $\beta$-actin. Lower panel: Relative Akt and mTOR expressions. A, control group; B, spinal cord injury (SCI) group; C, SCI and bone marrow stromal cells (BMSCs) transplantation group; D, SCI and BMSCs transplantation with treadmill exercise group. ${ }^{*} P<0.05$ compared to control group. ${ }^{\#} P<0.05$ compared to SCl group. ${ }^{\dagger} P<0.05$ compared to SCl and BMSCs transplantation group. 

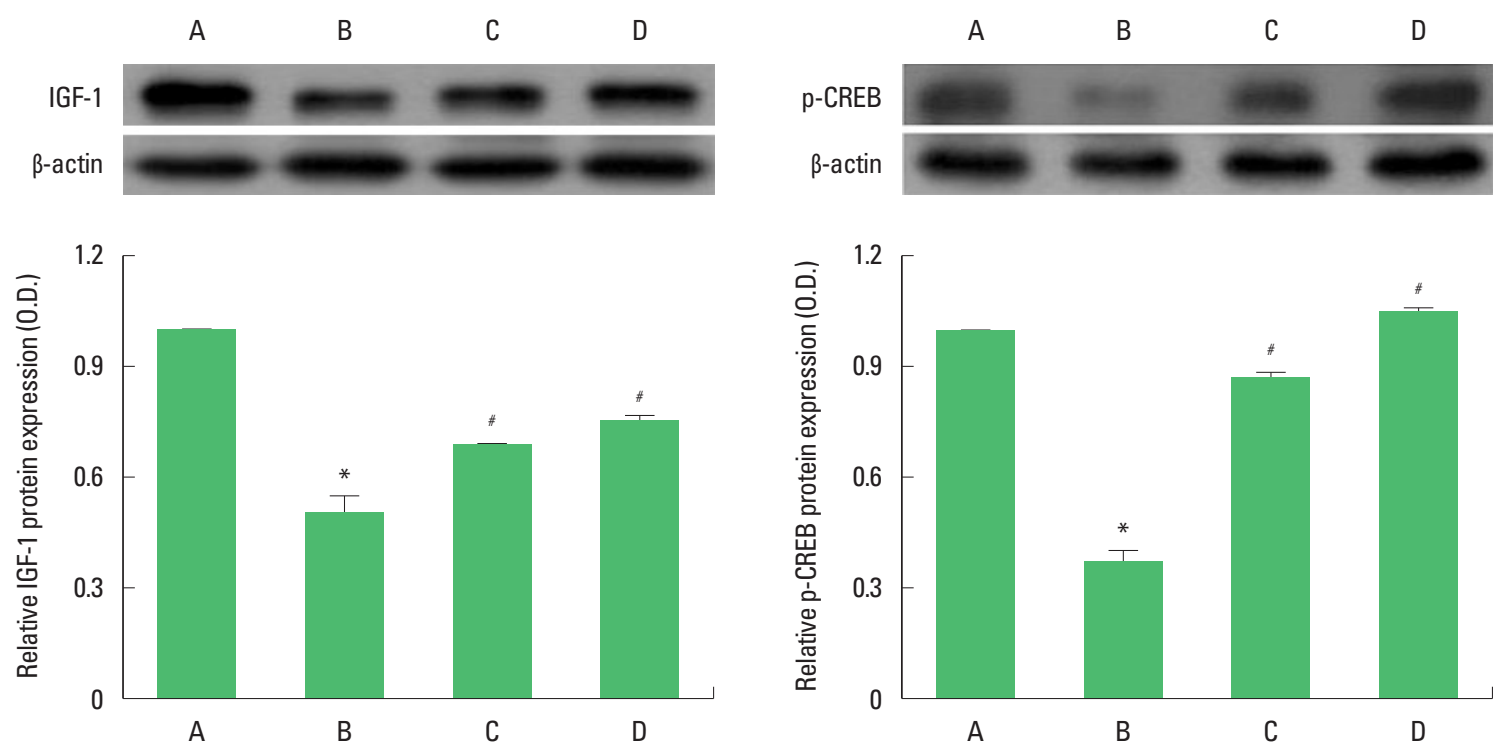

Fig. 3. Insulin-like growth factor-I (IGF-I) and phosphorylated cyclic adenosine monophosphate response element-binding protein (p-CREB) expressions in the soleus muscle. Upper panel: Representative expressions of IGF-I, p-CREB, and $\beta$-actin. Lower panel: Relative IGF-I and p-CREB expressions. A, control group; B, spinal cord injury (SCI) group; $\mathrm{C}, \mathrm{SCl}$ and bone marrow stromal cells (BMSCs) transplantation group; D, SCl and BMSCs transplantation with treadmill exercise group. ${ }^{*} P<0.05$ compared to control group. ${ }^{*} P<0.05$ compared to $\mathrm{SCl}$ group.

p-CREB, although statistical significance was not appeared.

\section{DISCUSSION}

To restore locomotor capacity after SCI, many researchers focused on restoring the lost neuronal cell and axonal sprouting around the spared spinal tissue, applying cell transplantation technique and regular exercise (Ide et al., 2010; Kim et al., 2017; Seo et al., 2013). But recent studies have emphasized that spinal cord regeneration can be successfully achieved when minimizing skeletal muscle atrophy with positive histological changes in the spinal nerves (Giangregorio and McCartney, 2006). Following SCI, cross sectional area of skeletal muscle decreases by $30 \%$ to $50 \%$, resulting from disuse and immobilization (Gorgey and Shepherd, 2010). The loss of muscle mass is induced by activation of myostatin (growth differentiation factor-8), which is an extracellular cytokine to inhibit muscle cell growth and differentiation.

In the present study, we examined expression level of myostatin in the soleus muscle after SCI and combination of treadmill exercise with BMSCs engrafting significantly decreased myostain compared to other groups.

Myostatin is an extracellular cytokine mostly expressed in skeletal muscles and known to play a crucial role in the negative regulation of muscle mass. Myostatin initiates several different signaling cascades resulting in the upregulation of the atrogenes and downregulation of the important for myogenesis genes (Elkina et al., 2011). Serum myostatin level in patients with SCI is statistically higher than those in healthy people (Invernizzi et al., 2015). Long et al. (2019) reported that blocking extracellular activation of myostatin provides a critical approach to increase protein synthesis in the skeletal muscle after SCI. For this reason, we thought that regular physical activity combined with BMSCs transplantation might be new therapeutic way to decrease muscle atrophy through activating skeletal muscle metabolism after SCI.

As mentioned above, these secondary changes of muscular system after SCI can increase the risk of metabolic disorders, such as osteoporosis, obesity, and diabetes (Chen and Stein, 2003). Several researchers studying on spinal cord regeneration have interested in the skeletal muscle hypertrophy in order to improve quality of life (Gorgey and Shepherd, 2010). IGF-1, belongs to IGF ligand, is highly correlated with the growth, development and hypertrophy of skeletal muscle (Velloso, 2008). Elkina et al. (2011) represent that IGF-I is inhibited when myostatin is overexpressed, and the absence of IGF-I increased apoptosis via upregulation of myostatin in C2C12 cells (Amirouche et al., 2009) as well as in muscle hypertrophy, IGF-I has been considered as a key protein to induce phosphatidyl inositol 3-kinase (PI3K), Akt, and mTOR signaling pathway. Retamales et al. (2015) suggested that myoblast differentiation and increase of cross-sectional area in skeletal muscle were facilitated by activating induce IGF-1/PI3K/Akt and mTOR 
signaling pathway.

In our study, we found that combination of treadmill exercise with BMSCs engrafting significantly up-regulated expression levels of IGF-I, Akt, and mTOR in soleus muscle after SCI. We speculate that exercise-mediated functional crosstalk between IGF-I downstream molecules and myostatin can suppress the apoptosis of satellite cells and atrophy in the muscle. Therefore, the present findings demonstrated the possibility that combining treadmill exercise with BMSCs transplantation might accelerate protein synthesis and hypertrophy in the soleus muscle after SCI through activation of IGF-I/mTOR signaling pathway.

\section{CONFLICT OF INTEREST}

No potential conflict of interest relevant to this article was reported.

\section{ACKNOWLEDGMENTS}

This study was supported by the National Research Foundation of Korea Grant funded by the Korean Government (NRF-2014S1A5B5A02013292).

\section{REFERENCES}

Amirouche A, Durieux AC, Banzet S, Koulmann N, Bonnefoy R, Mouret C, Bigard X, Peinnequin A, Freyssenet D. Down-regulation of Akt/ mammalian target of rapamycin signaling pathway in response to myostatin overexpression in skeletal muscle. Endocrinology 2009;150: 286-294.

Chen B. Stein A. Osteoporosis in acute spinal cord injury. Top Spinal Cord Inj Rehabil 2003;9:26-35.

DePaul MA, Lin CY, Silver J, Lee YS. Combinatory repair strategy to promote axon regeneration and functional recovery after chronic spinal cord injury. Sci Rep 2017;7:9018.

Elkina Y, von Haehling S, Anker SD, Springer J. The role of myostatin in muscle wasting: an overview. J Cachexia Sarcopenia Muscle 2011;2: 143-151.

Giangregorio L, McCartney N. Bone loss and muscle atrophy in spinal cord injury: epidemiology, fracture prediction, and rehabilitation strategies. J Spinal Cord Med 2006;29:489-500.

Gorgey AS, Shepherd C. Skeletal muscle hypertrophy and decreased intramuscular fat after unilateral resistance training in spinal cord injury: case report. J Spinal Cord Med 2010;33:90-95.

Ibitoye MO, Hamzaid NA, Hasnan N, Abdul Wahab AK, Davis GM.
Strategies for rapid muscle fatigue reduction during FES exercise in individuals with spinal cord injury: a systematic review. PLoS One 2016;11:e0149024.

Ide C, Nakai Y, Nakano N, Seo TB, Yamada Y, Endo K, Noda T, Saito F, Suzuki Y, Fukushima M, Nakatani T. Bone marrow stromal cell transplantation for treatment of sub-acute spinal cord injury in the rat. Brain Res 2010;1332:32-47.

Invernizzi M, Carda S, Rizzi M, Grana E, Squarzanti DF, Cisari C, Molinari C, Renò F. Evaluation of serum myostatin and sclerostin levels in chronic spinal cord injured patients. Spinal Cord 2015;53:615-620.

Jung SY, Seo TB, Kim DY. Treadmill exercise facilitates recovery of locomotor function through axonal regeneration following spinal cord injury in rats. J Exerc Rehabil 2016;12:284-292.

Kim YM, Jin JJ, Lee SJ, Seo TB, Ji ES. Treadmill exercise with bone marrow stromal cells transplantation facilitates neuroprotective effect through BDNF-ERK1/2 pathway in spinal cord injury rats. J Exerc Rehabil 2018;14:335-340.

Kim YM, Seo TB, Kim CJ, Ji ES. Treadmill exercise with bone marrow stromal cells transplantation potentiates recovery of locomotor function after spinal cord injury in rats. J Exerc Rehabil 2017;13:273-278.

Long KK, O'Shea KM, Khairallah RJ, Howell K, Paushkin S, Chen KS, Cote SM, Webster MT, Stains JP, Treece E, Buckler A, Donovan A. Specific inhibition of myostatin activation is beneficial in mouse models of SMA therapy. Hum Mol Genet 2019;28:1076-1089.

Oudega M, Xu XM. Schwann cell transplantation for repair of the adult spinal cord. J Neurotrauma 2006;23:453-467.

Retamales A, Zuloaga R, Valenzuela CA, Gallardo-Escarate C, Molina A, Valdés JA. Insulin-like growth factor-1 suppresses the Myostatin signaling pathway during myogenic differentiation. Biochem Biophys Res Commun 2015;464:596-602.

Seo TB, Cho HS, Shin MS, Kim CJ, Ji ES, Baek SS. Treadmill exercise improves behavioral outcomes and spatial learning memory through up-regulation of reelin signaling pathway in autistic rats. J Exerc Rehabil 2013;9:220-229.

Shin MS, Park HK, Kim TW, Ji ES, Lee JM, Choi HS, Kim MY, Kim YP. Neuroprotective effects of bone marrow stromal cell transplantation in combination with treadmill exercise following traumatic brain injury. Int Neurourol J 2016;20(Suppl 1):S49-56.

Thompson R, Sakiyama-Elbert S. Using biomaterials to promote pro-regenerative glial phenotypes after nervous system injuries. Biomed Mater 2018;13:024104.

Tian Y, Liu X, Jia M, Yu H, Lichtner P, Shi Y, Meng Z, Kou S, Ho IHT, Jia B, Cheng BCP, Lam CKM, Tsang S, Wong SH, Yu J, Cheng CHK, Gin T, Wu WKK, Chen Z, Chan MTV; Persistent pain after surgery study investigators. Targeted genotyping identifies susceptibility locus in 
brain-derived neurotrophic factor gene for chronic postsurgical pain. Anesthesiology 2018;128:587-597.

Tu WZ, Li SS, Jiang X, Qian XR, Yang GH, Gu PP, Lu B, Jiang SH. Effect of electro-acupuncture on the BDNF-TrkB pathway in the spinal cord of CCI rats. Int J Mol Med 2018;41:3307-3315.

Velloso CP. Regulation of muscle mass by growth hormone and IGF-I. Br
J Pharmacol 2008;154:557-568.

Wheaton BJ, Noor NM, Whish SC, Truettner JS, Dietrich WD, Zhang M, Crack PJ, Dziegielewska KM, Saunders NR. Weight-bearing locomotion in the developing opossum, Monodelphis domestica following spinal transection: remodeling of neuronal circuits caudal to lesion. PLoS One 2013;8:e71181. 